\title{
YouTube Video Recommendation via Cross-Network Collaboration
}

\author{
Tejal T. Wanave \\ PG Student \\ Department of Computer Engg. \\ V.P.C.O.E.Baramati, \\ Pune, India
}

\author{
Sheetal A. Takale \\ Assistance Professor \\ Department of Computer Engg. \\ V.P.C.O.E.Baramati, \\ Pune, India
}

\begin{abstract}
We present a system that automatically recommends videos to the three YouTube users: new, light and heavy. We have proposed YouTube video cross network recommendation system; it extracts users auxiliary information on Twitter to address the three typical problems: new user, cold start and sparsity which are occurring in single network recommendation system. At the first stage, the system recommends videos to the new user using cross relevance method, it maps individual user Twitter preferences with video titles on YouTube. Our system has calculated a tweet vocabulary of more than 400 words using Hash Map function. At the second stage, we construct textual and visual similarity between user's data on different OSNs (Online Social Networks), i.e. the system recommends videos to light and heavy users by considering their data on both Twitter and YouTube. Here we map visual based features of useruploaded videos on YouTube with all other videos in a database also uploaded video titles is used to automatically suggest relevant videos on the basis of text similarity. These methods give benefit to new, light and heavy users who are having limited or fr++++

equent behavior record on YouTube. Finally, we compared our cross-relevance method with other single network based methods a) the average relevance of videos automatically recommended by our system for new YouTube users is $76 \%$ with Top $\mathrm{K}=5$. b) The average relevance of videos automatically recommended by our system for light and heavy YouTube users is $90 \%$ with Top $\mathrm{K}=5$.
\end{abstract}

\section{General Terms}

Tweets, Videos, Word Similarity, Text similarity algorithm, Visual similarity algorithm, New user, Light user, Heavy user.

\section{Keywords}

YouTube video recommendation, Cross-network social relevance, Matrix factorization, User Modeling, Online social network.

\section{INTRODUCTION}

Social media has changed the way people share and access information. With social media services, users necessarily interact with each other in social networks [5] [1]. People on Facebook may communicate with their friends, follow realtime hot events on Twitter and subscribe videos on YouTube, so these cross -network user activities identify users interest and their ever-changing preferences.

YouTube recommendation system is based on the single online social network. This system recommends videos to the user based on their interest, by identifying users recent activities on YouTube [1]. User available data on one online social network is not sufficient to understand their interest and capture their changing preferences. This is the time when a new, cold start and sparsity problems start [7]. YouTube video recommendation system suffers from different problems such as Recommendation to a new user, cold start and sparsity. Also trust, scalability, privacy, and positive rating, Types of users are shown in figure 1 .

New user -A user newly registered on YouTube with zero historical behavioral records on the target network. In this case recommended systems are unable to give a recommendation [2]. Cold start -This problem occurs when user has a lack of historical data or limited historical behavioral records on the target network. Such users are called light users. In this situation, recommended system is unable to provide accurate recommendations [3]. Sparsity - It arises when the number of items and users are present and when user gives rating to some of the items. In such case, the user item interaction matrix is very sparse and recommended systems is not able to provide accurate recommendations for such users who are having frequent interaction in the target network [7].

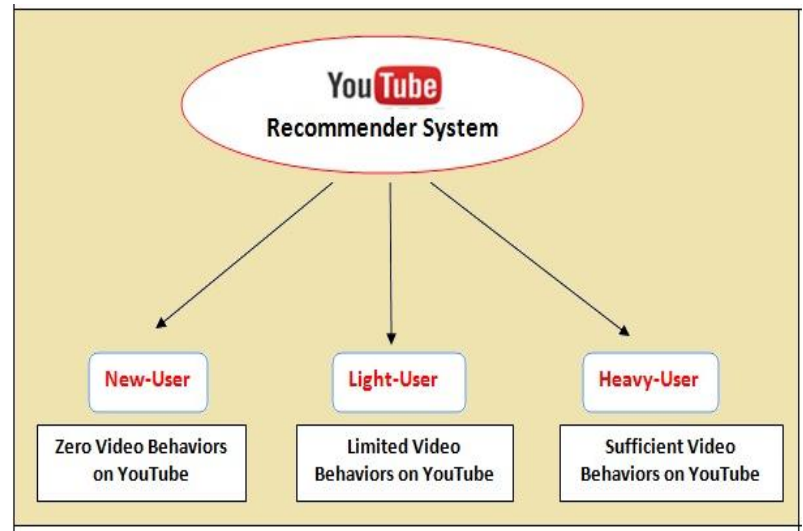

Figure 1: Problems in YouTube recommendation System.

Trust - It occurs when a user with the limited amount of historical behavioral data on the social network is not relevant as compared to the user having a large number of historical data. Scalability- On the internet or web, the number of users and items are increasing rapidly so we need to filter data according to user's efficiency. Most systems require a lot of resources to handle this data. Privacy - The data which user makes private on their account cannot be extracted and it violates the user's privacy. To address these above-mentioned problems, our work is motivated to access users scattered data in multiple online social networks to improve personalized 
recommendation in the target network i.e. user's rich crossnetwork activity data is used to identify video preferences on YouTube and design a cross-network YouTube video recommendation solution [4].

The motivation behind this research work is to understand the problems in recommended systems. Earlier single network based recommended systems used to deal with one or two of the problems. A Cross network video recommendation solution simultaneously addresses all problems in the single network recommendation system and gives benefit to three types of YouTube users, by extracting the user rich crossnetwork activity data from twitter, Google+ and YouTube. Also, by extracting user data from these different auxiliary networks, it helps to understand the user's interest and their changing preferences. Finally, the developed cross relevance fusion method helps to address the challenges which are occurring in cross-network video recommendation [5] [8].

The remaining paper is structured as follows. Section 2 surveys related works on social network recommendation systems. Through section 3, 4 and 5, we describe different components of the proposed system. Section 6,shows experimental results and analysis. Finally, we conclude the paper in section 7 .

\section{LITERATURE SURVEY}

Many studies and analysis have been performed on Recommendation systems.

A. Behavioral-modeling approach MOBIUS methodology [3] In this, a Behavioral-modeling approach: Connecting user's across social media sites. MOBIUS (modeling, behavioral information of user across sites) Methodology is used to connect users across communities and it helps to address the major problem of user cross linking.

B. personalized video recommendation using cross platform user modeling [4] In this, system proposed a personalized video recommendation solution based on crossplatform user modeling. Optimal combination and Cross platform modeling used to find personalized video recommendation on YouTube by transferring users behavioral activity on auxiliary network i.e. Google+. This system is based on two strategy 1.profile enrichment 2.Relationship transfer. In the first profile enrichment stage users demographic information is transferred to recommend videos in target platform and cross platform modeling, it helps to identify users behavioral records

on different OSNs, and based on that video recommendation is done on the target network. Also Proposed valuable method is tackled the sparsity and the cold start problem issue.

\section{Social transfer model for cross-domain transfer} learning [5] In this, a Social transfer model is used to identify cross domain transfer learning. Use real time social streams to build mutual connection between social media by using an online streaming LDA (Latent Dirichlet Allocation) model which is used to learn topic space in real time and Social transfer model is used to incorporate learned topic model from social networks into the transfer leaning framework.

\section{Recommender system with social regularization[8]}

This approach performs other art-of-the state methods when do analysis on large datasets. This method extracts the contextual information from social network, i.e. users social tags. The matrix factorization framework is used to improve the prediction accuracy of recommender system.
E. Cross-system user modeling approach on the social web [9] In this The Generic recommendation algorithm used to generate more valuable tag based profiles. The system considers users tag based profiles, which are created from users tagging activity in flicker, twitter. And identify at what extent users tag based profile overlap on the different OSNs. The result gives 10 times higher precision for recommendation and address the cold start problem.

F. cold-start problem in recommender systems with social tags [10] Diffusion based recommendation Algorithm is used to improve diversity of recommendation and solve problems in social tagging. Including social tag could be some extents, it helps to solve cold start problem in recommendation system.

G. YouTube video recommendation system[11] System provides sets of videos to user's who having their account on YouTube site. Based on user's previous activity on the YouTube site, system gives personalised Recommendations, are featured in two primary locations: The YouTube home page (http://www.youtube.com) and at the Browse page http://www.youtube.com/videos. A Co visitation based graph of videos is created to analyze users recent activities on YouTube.

H. Multifaceted collaborative filtering model [12] Yehuda Koren [13] proposed a Factorization which meets the neighborhood multifaceted collaborative filtering model and neighborhood latent factor model. The nearest neighbor method recommends an item to the user based on users profile. The system recommends item to user based upon the description of item profile of the user. In this user item interaction matrix is created. The system achieves more accuracy by combining these two methods, i.e. collaborative filtering, and latent factor model. The model achieves more accuracy, improve predictability, and handle new user without training. Recommendation Challenges: System faces two challenges while performing recommendation by using crossplatform information. (1) User data on different online social networks is heterogeneous, so we cannot directly utilize users tweet data, collected from twitter to identify user video preference on YouTube. (2) For the light and heavy user the estimated preference on Twitter, Google+, and observed behavior on YouTube may contradict with each other.

\subsection{Table in brief}

Table 1: Summary of Literature Survey

\begin{tabular}{|l|l|l|}
\hline Authors & Techniques Used & Algorithm/Method \\
\hline $\begin{array}{l}\text { Zi-Ke Zhang } \\
{[11]}\end{array}$ & $\begin{array}{l}\text { Tag clustering } \\
\text { technique }\end{array}$ & $\begin{array}{l}\text { Diffusion } \\
\text { Algorithm }\end{array}$ \\
\hline Fabian Abel [10] & user modeling & $\begin{array}{l}\text { Generic } \\
\text { recommendation }\end{array}$ \\
\hline Hao ma [9] & $\begin{array}{l}\text { Missing Data } \\
\text { predication }\end{array}$ & Matrix Factorization \\
\hline $\begin{array}{l}\text { Suman Deb Roy } \\
\text { [6] }\end{array}$ & $\begin{array}{l}\text { Media } \\
\text { Recommendation }\end{array}$ & $\begin{array}{l}\text { LDA, Social transfer } \\
\text { algorithm }\end{array}$ \\
\hline $\begin{array}{l}\text { Zhengyu Deng } \\
\text { [5] }\end{array}$ & $\begin{array}{l}\text { Video } \\
\text { recommendation }\end{array}$ & Optimal combination. \\
\hline $\begin{array}{l}\text { Huan Liu [4] } \\
\text { User } \\
\text { Identification }\end{array}$ & $\begin{array}{l}\text { MOBIUS } \\
\text { Methodology }\end{array}$ \\
\hline Mi Zhang [3] & $\begin{array}{l}\text { Cross-OSN } \\
\text { analysis }\end{array}$ & $\begin{array}{l}\text { supervised Co- } \\
\text { training Algorithm }\end{array}$ \\
\hline Jita Sang [1] & Cross-relevance & Matrix Factorization \\
\hline
\end{tabular}




\section{OVERVIEW OF OUR SYSTEM}

Our proposed system performs hybrid recommendation to address these above challenges; it considers both textual and visual content data to recommend videos to three typical YouTube users, as shown in Fig. 2.

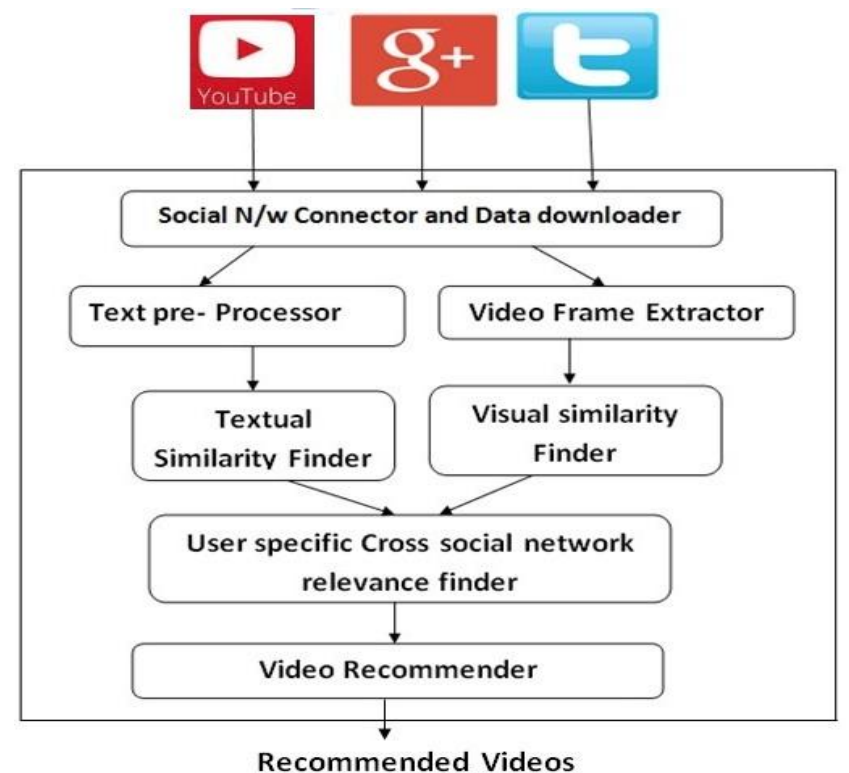

Figure 2:- system architecture for cross network video recommendation.

The system consists of five main steps, as follows: (a) Social Network API; (b) Text pre-processing; (c) Textual similarity Finding; (d) Visual Similarity Finding; and (e) Cross-Platform Relevance. Initially, we download the users data from their Twitter and YouTube accounts by using Twitter API and YouTube API respectively, i.e. download, upload, favorite and added playlist Videos and their titles on YouTube, recent tweets on Twitter. Our proposed system identifies cross relevance between tweets on twitter with video and their titles on YouTube [6]. Based on this information, system recommends videos to the users on YouTube. The System uses Latent Dirichlet Allocation for topic separation and the matrix Factorization model to identify latent space discovery i.e. user-video preferences.

\subsection{Pre-processing}

Our proposed video recommendation system is based on matrix factorization method. It maps both users and videos to their latent factor space and user-video interaction matrix is created. To avoid over fitting error, MF model suggests discovering the latent structure based only on the observed interactions.

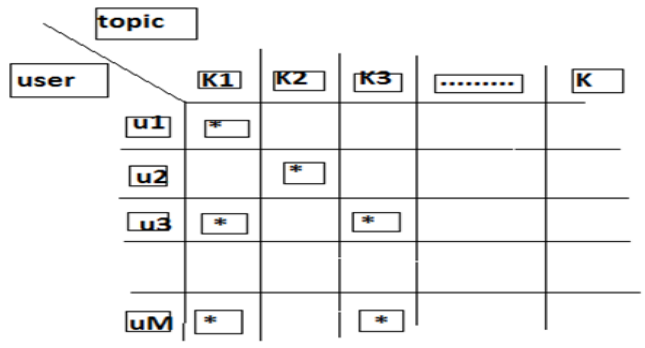

Figure 3 : User-Topic relation on Twitter

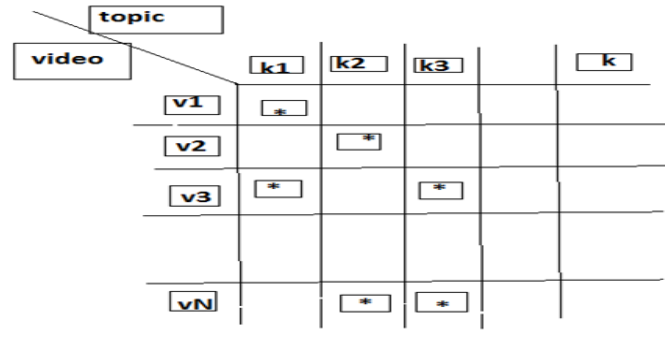

Figure 4 : Video-Topic relation on YouTube

Where, U - (set of overlapped users), V - (set of video interacted). $\mathrm{U}=\{\mathrm{u} 1, \mathrm{u} 2, \mathrm{u} 3, \cdots, \mathrm{uM}\} \in \mathrm{R}^{\wedge} \mathrm{M} \times \mathrm{K} . \mathrm{V}=$ $\{\mathrm{v} 1, \mathrm{v} 2, \mathrm{v} 3 . \cdot, \mathrm{vN}\} \in \mathrm{R}^{\wedge} \mathrm{N} \times \mathrm{K}$, are the user and video representations in the K-dimension latent space, as shown in figure 3,4. We have to find a user-video relationship matrix $(\mathrm{Y})$, such that $(\mathrm{M} \times \mathrm{K}) \times(\mathrm{N} \times \mathrm{K})^{\wedge} \mathrm{T}=(\mathrm{M} \times \mathrm{K}) \times(\mathrm{K} \times \mathrm{N}), \mathrm{Y}=$ $\mathrm{R}^{\wedge}(\mathrm{M} \times \mathrm{N})$, matrix $\mathrm{Y}$ record the observed user-video entries from this we can directly estimate the user ui's preference on video $\mathrm{vj}$, as shown in figure 5 . To reduce the influence of sparsity, videos with similar content should have close representations in the derived latent factor space.

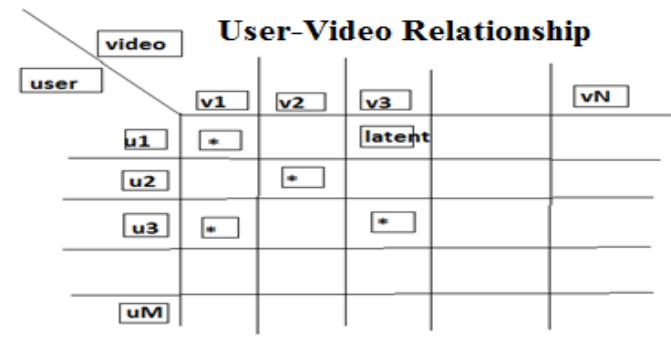

Figure 5 : User-Video relation on YouTube.

In summary, our main contribution to the existing work includes:

A Cross-network video recommendation system, It considers users data on both Twitter and YouTube to identify their video preferences on YouTube, which improve the accuracy of recommendation system.

Our system addresses the problems that YouTube suffers from such as, New-user, Cold-start and Data-sparsity.

For new user, system considers their post on Twitter to identify his/her video preferences on YouTube.

For light and heavy users, system considers their text and visual similarity data on both Twitter and YouTube to identify his/her YouTube video preferences.

\subsection{Processing}

System is divided into four phases: System $=\{F$ Collection, $\mathrm{F}$ Categorization $\mathrm{F}$ Classification, $\mathrm{F}$ Comparison, $\mathrm{F}$ Recommendation \} as shown in below figure 6 .

F Collection $=$ Collect textual content $($ Tweets from twitter, profile from Google+), visual content (Videos from YouTube)

F categorization $=$ grouping similar textual and visual content as different topic space.

F classification $=$ Classify data in the user-topic and videotopic relationship, latent space matrix created

(a) Video topic similarity Finding. $Q=\mathrm{R}^{\wedge}\left(\mathrm{N}^{*} \mathrm{~K}\right)$

(b) User topic similarity Finding. $\mathrm{P}=\mathrm{R}^{\wedge}\left(\mathrm{M}^{*} \mathrm{~K}\right)$ 
F comparison $=$ Get User-video relationship matrix. Finding cross -relevance by comparing similarity between textual data on twitter with visual content on YouTube

\section{User-Video similarity Finding. $\mathrm{Y} \| \mathrm{R}^{\wedge}\left(\mathrm{M}^{*} \mathrm{~N}\right)$}

F Recommendation $=$ Based on this similarity value system recommend videos to the three YouTube users, i.e. New, Light, Heavy. The existing works proposes to deal with one or two of the problems, but a YouTube video recommendation solution via cross-network collaboration addresses all the three problems simultaneously.

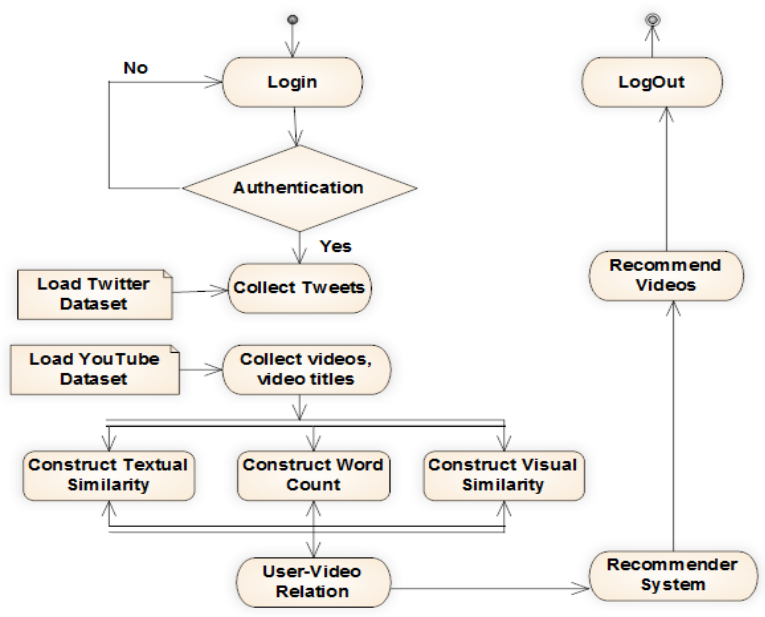

Figure 6: System Flow

\section{CROSS-NETWORK VIDEO RECOMMENDATION SOLUTION}

Cross-network recommendation system considers both twitter and YouTube OSNs and uses textual and visual similarity methods to recommends videos to three YouTube users; new, light, and heavy. It also addresses the recommendation problems which have occurred in earlier recommended systems.

\subsection{Text Pre-processing}

The tweets may contain a misspelling, acronyms, and even the interpretation is also ambiguous. For understanding the exact meaning of such data, we need to remove noise from tweets. Following are the text preprocessing steps [4].

1. Tweets may contain slang words, e.g. omg, gn. We replace slangs by their standard forms by using the slang word dictionary

2. Tweets containing words with consecutive repeating letters, e.g. yesssss, hahaha. We replace them by one letter so, original word remains as it is. We can recognize such words such words by using regular expressions.

3. Remove all non-letter symbols and punctuations

\subsection{Construct Textual Similarity}

We have performed text preprocessing on textual data, i.e. porter stemmer and stop word remover class in the weka.jar file is used to perform stemming and remove stop words from text data, e.g. Tweets, video titles. After the textual preprocessing we get the data in the form of tokens. At the last stage, we calculate the similarity between each tweet with all other tweets using the cosine similarity distance formula.
(Ti. $\mathrm{Tj}$ )

$$
\text { Cosine similarity }(\mathrm{Ti}, \mathrm{Tj})=\frac{}{(\|\mathrm{Ti}\| \cdot\|\mathrm{Tj}\|)}
$$

Where Ti, Tj: Tweet documents.

To find similarity between each tweet (i.e. consider as a one text document) with all other tweets, we first calculate the TfIDf score for each document, using Equation (2) [(Tf-IDf) term frequency-Inverse document frequency].

Term Frequency $=($ word, $\operatorname{doc} 1)$

$$
\mathbf{T F}=\left[\frac{\text { No of times word occurs in that document }}{\text { Total number of words in that document }}\right]
$$

Inverse Document Frequency $=($ word, docs $)$

IDf $=1+\operatorname{loge}\left[\frac{\text { Total number of documents }}{\text { Number of times word occur in that document }}\right]$

After this by using Tf-IDf score cosine similarity values are calculated for each tweet document by using Equation (1), these values give the distance between each document with all other documents. Based on these values of cosine similarity we identified how each document is similar or different with other documents.

Tweet 0 - Beauti natur

Tweet 1 - Diet veget soup video love it

Tweet 2 - Veget corn soup video

Tweet 3 - Chocol spong cake video

Tweet 4 - Coffe chocol cake tast

Tweet 5 - Toothpick nail art fashion video

Here after preprocessing how these 5 tweets are similar to each other by using cosine similarity are shown in figure 6 .

$\begin{array}{rcrrrrr} & \mathrm{T} 0 & \mathrm{~T} 1 & \mathrm{~T} 2 & \mathrm{~T} 3 & \mathrm{~T} 4 & \mathrm{~T} 5 \\ \mathrm{~T} 0 & \mathbf{1 . 0} & \mathbf{0 . 4 3} & \mathbf{0 . 0 4} & \mathbf{0 . 0} & \mathbf{0 . 0 4} & \mathbf{0 . 0 3} \\ \mathrm{T} 1 & \mathbf{0 . 4 3} & \mathbf{1 . 0} & \mathbf{0 . 0 6} & \mathbf{0 . 0} & \mathbf{0 . 0 6} & \mathbf{0 . 0 5} \\ \mathrm{T} 2 & \mathbf{0 . 0 4} & \mathbf{0 . 0 6} & \mathbf{0 . 9 9} & \mathbf{0 . 0} & \mathbf{0 . 0 6} & \mathbf{0 . 0 5} \\ \mathrm{T} 3 & \mathbf{0 . 0} & \mathbf{0 . 0} & \mathbf{0 . 4 3} & \mathbf{1 . 0} & \mathbf{0 . 0} & \mathbf{0 . 0} \\ \mathrm{T} 4 & \mathbf{0 . 0 4} & \mathbf{0 . 0 6} & \mathbf{0 . 0 6} & \mathbf{0 . 0} & \mathbf{1 . 0} & \mathbf{0 . 5 5} \\ \mathrm{T} 5 & \mathbf{0 . 0 3} & \mathbf{0 . 0 5} & \mathbf{0 . 0 5} & \mathbf{0 . 0} & \mathbf{0 . 5 5} & \mathbf{1 . 0}\end{array}$

Figure 7: Tweet similarity matrix

To construct tweet similarity, we also build a topic vocabulary after removing stop word and non-words. Each topic is used to build a user preference, i.e. the topics on which user is having maximum interest.

\footnotetext{
Algorithm 1: Text Similarity Algorithm

Require: User account Tweets $\mathrm{Ti}=\mathrm{t} 1, \mathrm{t} 2, \ldots \mathrm{tn}$.

Result: Number of similar tweet documents T.

Initialization: $\mathbf{T}=\Phi$

For Each tweet do
} 
1) Perform text preprocessing and store each tweet (Ti) in a one text document.

2) Get the document identifiers id1, id2....idn of the tweet files in the index.

3) Calculate Tf-IDf score of all tweet documents (Ti) and store as vectors $\mathrm{v} 1, \mathrm{v} 2, \ldots \ldots \mathrm{vn}$ and print them.

$\mathrm{Tf}=$ word $/ \mathrm{t} 1 \quad \mathrm{IDf}=\mathrm{Ti} /$ word

4) Calculate cosine similarity values between t1 to th using eq. (1) and print them.

If (value $>=0.6$ ) then similar $\mathrm{t} 1$ and $\mathrm{t} 2$.

\section{End for}

\section{Return T.}

\subsection{Calculation of Word Frequency Count}

The system calculates word count in each tweet document, by using hash map function, i.e. Hashing. Hash all words one by one in the hash table. If a word is already present, then increment its count. Finally, traverse through the hash table and return the Top k-word with a maximum count.

Here we have taken Top 5 and Top 3 tweet words with maximum word count after which we match those words with all video titles on YouTube. And finally one or more matching word related videos on the basis of content matching are recommended to the users on YouTube.

\subsection{Construct Visual Similarity}

To find out the similarities between the videos initially, we have completed each video frame extraction by using IMediaListener.onVideoPicture method from the xugglerXuggler-5.4.Jar library. Here we get the extracted frame images in the BGR 24bit color format. After this, we extract each video key frame 135 features and store it in database for video comparison purpose.

Here are the features which we consider for video comparison purpose:

Autocorrelation - 25 features

Average RGB - 03 features

Color Moment - 20 features

Co-occurrence - 20 features

Edge Directional Histogram 72 features

Geometric Moment 01 feature

Invariant Moment - 05 features

we consider each video key-frame features with distance $=3$ to perform matching between relevant videos, i.e. every $3 \mathrm{rd}$ frame feature of each video is compared with all other video subsequent frame features; the cosine similarity distance formula in Equation (1) is used to compare each video frames feature with all other video frame features.

\section{Algorithm steps}

1. Read the input video name.

2. Read all frames of the input video.
3. Calculate key frames 135 feature such as text, color, and moment.

4. Finally, compute the feature vectors for all key frames.

5. Load features of the each video key-frames into a database.

6. Compare the difference between each video key frame feature and the features of the database video key frames.

7. Calculate the closest distance of the features of the key frames in a database by using cosine similarity.

8. Retrieves the input video matching videos from the database.

9. Display the similarity between videos in the output panel.

\author{
Algorithm 2: Visual Similarity Algorithm \\ Require: User uploaded videos $\mathrm{Vi}=\mathrm{v} 1, \mathrm{v} 2, \ldots$ vn. \\ Result: Number of similar videos V. \\ Initialization: $\mathbf{V}=\Phi$ \\ For Each video do \\ 1. If ( Vi C.AVI media format) then. \\ 2. Compute the frame extraction $(\mathrm{F})$ of each video file. $\mathrm{v} 1$ \\ $=\{\mathrm{F} 11, \mathrm{~F} 12, \mathrm{~F} 13 \ldots \mathrm{F} 1 \mathrm{n}\}$ and $\mathrm{v} 2=\{\mathrm{F} 21, \mathrm{~F} 22, \mathrm{~F} 23 \ldots \mathrm{F} 2 \mathrm{~N}\}$ \\ 3. Calculate key frames of each video file. $\mathrm{Fk}=2$ distance.
} $\mathrm{Fk}=\{\mathrm{F} 12, \mathrm{~F} 14, \mathrm{~F} 16 .$.$\} and so on.$

For Each key frame do

4. Compute the 135 features (f) $\mathrm{Fk}=\{\mathrm{fl}, \mathrm{f} 2, \mathrm{f} 3 \ldots \mathrm{fn}\}$

5. If (features value $=135$ ) then create a feature vector.

6. Compute the cosine similarity distance. If value(Threshold) $>=0.6$ then similar $\mathrm{v} 1$ and $\mathrm{v} 2$.

End For.

Return V.

\section{USER CROSS-RELEVANCE}

To find the user cross relevance data, we must first download the number of users tweets on their twitter account and also the videos and video titles on YouTube. At this stage we get the text and a video similarity matrix, i.e. number of tweets and videos on different topic space. Now, we map these users preferences on twitter with video contents on YouTube by using matrix factorization [11]. 
Matrix Factorization: How to calculate cross relevance between user's data on the Different OSNs by using matrix factorization method is shown below. Here $R$ is the users observed video interaction matrix on YouTube and $\mathrm{R}^{*}$ is the estimated user's video preferences matrix on YouTube [12]. Our goal is to calculate $\mathrm{R}^{*}$ such that it is approximately similar to users real video preference on YouTube.

The system performs video recommendation on the basis of ranking, i.e. Maximum user interested videos are recommended, by analyzing users recent activities from their twitter, YouTube, Google+ account. Here video recommendation is made for new user, light, and heavy YouTube users

\section{Algorithm 3: Cross Relevance Algorithm}

Require: User, Video topics matrix P, Q.

Result: User - video preference matrix $\mathrm{R}^{*} \approx \mathrm{R}$

Initialization: $\mathbf{R}^{*}=\Phi$

For Each P and Q do

1. To find $\mathrm{P}(\mathrm{a}|\mathrm{U}| \times \mathrm{k}$ matrix $)$ and $\mathrm{Q}(\mathrm{a}|\mathrm{V}| \times \mathrm{k}$ matrix $)$ such that $\mathrm{R} \approx \mathrm{P} \times \mathrm{Q}^{\wedge} \mathrm{T}=\hat{\mathrm{R}}$

2. $\quad$ rij $=$ pi $^{\wedge}$ T.qj $($ Calculate dot product $)$

3. Find Stochastic gradient decent : $\mathrm{e}^{\wedge} 2(\mathrm{ij})=\left(\mathrm{rij}-\mathrm{r}^{*} \mathrm{ij}\right)$

$$
\begin{aligned}
& =(\text { rij }-\Sigma(\mathrm{k}=1 \text { to } \mathrm{k}) \text { Pik.qkj })^{\wedge} 2 \\
& =-2\left(\mathrm{rij}-\mathrm{r}^{*} \mathrm{ij}\right)(\mathrm{qkj})=-2 \mathrm{eij} \mathrm{qkj} \\
& =-2\left(\mathrm{rij}-\mathrm{r}^{*} \mathrm{ij}\right)(\mathrm{qik})=-2 \mathrm{eij} \text { qik }
\end{aligned}
$$

4. Apply update rule:

$$
P^{\prime}{ }^{i k}=P^{2} k+2 \alpha \text { eij qkj q' kj }=q k j+2 \alpha \text { eij Pik }
$$

\section{End For}

Return R*.

\subsection{Video Recommendation}

\subsubsection{New User}

When newly registered user on the YouTube, a user with zero historical behavioral records on the target network. For such a user most of the single network based recommendation system unable to identify user interest. So the Cross-network

YouTube video recommendation system is designed. The system considers overlapped users to recommend videos to three typical YouTube users [9] [10].

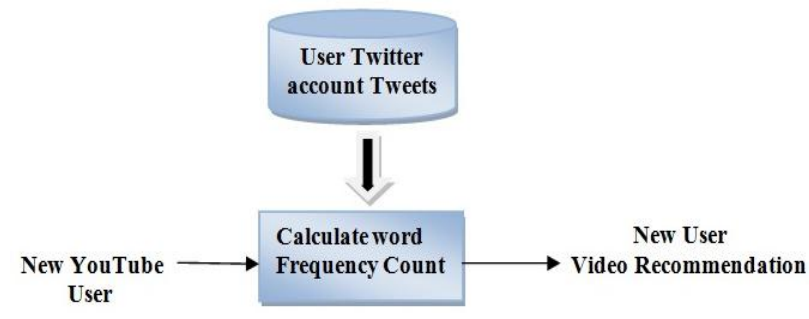

Figure 8: New user video recommendation
1. At first system downloads user tweets on their own twitter account. After downloading all tweets, text preprocessing removes stop-words, and stores each tweet in one text document.

2. Next Hash Map function is used to count word frequency in each tweet text document. Now from all tweet words count we select the Top five (5) words with maximum count. These words are nothing but the users maximum interest we found on their own twitter account.

3. Finally, these words are compared with video titles on YouTube. Then matching related videos are recommended to the user who does not have any behavior on YouTube, shown in figure 8 .

\subsubsection{Light and Heavy User}

To recommend videos to light and heavy users, we consider users data on Twitter and YouTube. These users show some behaviors on YouTube also, shown in Fig. 9

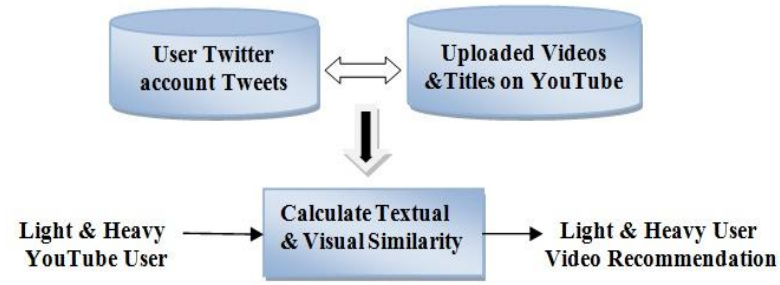

Figure 9: Light and heavy user video recommendation

1. We first consider users YouTube data, i.e. uploaded or favorite or added to playlist videos on YouTube, and compare these videos with all other videos in the database based on video frame features and matching videos are recommended to the users.

2. These recommended videos are matched again with all other videos on the video titles using cosine similarity and recommend matching videos to these users.

\section{DATASET}

The user shares their user accounts on different OSNs in the Google+ Profile. We have collected the 25 Google+ users who provide their user account on both YouTube and Twitter. We have further examined those users on YouTube and Twitter Via the respective APIs and crawled data of these 25 users who are publicly accessible and have behaviors on the both OSNs. These 25 users are recorded as the overlapped users. Specifically, on YouTube, for each of the overlapped users, we have downloaded their uploaded videos. For each video, the video tags, titles are also collected. On Twitter, for each user, we have downloaded their recent more than 70 tweets. While creating a dataset of Twitter, System has to visit developers' page of Twitter and it has to request an API key.

\section{Performance metrics:}

We select Recall, Precision, and F1 as the evaluation metrics.

Recall $=$ No. of relevant recommended videos $/$ No. of relevant videos.

Precision=No. of relevant recommended videos / No. of. retrieved recommended video.

F1 = 2.Precision.Recall $/$ Precision + Recall 


\section{K-Value Parameter Setting}

$\mathrm{K}$ is the parameter used in the Cross-platform relevance model. We calculate the number of top five videos on YouTube and it is set to $\mathrm{K}=5$ and $\mathrm{K} 3$ recommendation for testing purpose and found that it is giving relevant personalize recommended videos for Top $=5$, which accurate for Cross network video recommendation, shown in Fig 10.

\begin{tabular}{|c|c|c|c|c|c|}
\hline Users & Tweet Collection & $\begin{array}{l}\text { Matching } \\
\text { Tweets }\end{array}$ & $\begin{array}{c}\text { Retrieved } \\
\text { Recommended } \\
\text { Videos }\end{array}$ & $\begin{array}{c}\text { Relevant } \\
\text { Recommended } \\
\text { Videos }\end{array}$ & Precision \\
\hline 5 & $\begin{array}{l}\text { Vegetable Corn soup video, } \\
\text { Chocolate Sponge cake 1td } \\
\text { Coffee And Chocolate Cake, } \\
\text { Tooothpicks nail art tashion } \\
\text { Neil art blue design fashio } \\
\text { The color Nail Polish fashi }\end{array}$ & $\begin{array}{l}\text { Top }=5 \\
\text { Top }=3\end{array}$ & & $\begin{array}{l}19 \\
13\end{array}$ & $\begin{array}{l}0.76 \\
0.65\end{array}$ \\
\hline 10 & 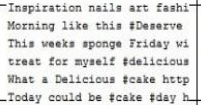 & $\begin{array}{l}\text { Top }=5 \\
\text { Top }=3\end{array}$ & & $\begin{array}{l}11 \\
8\end{array}$ & $\begin{array}{l}0.68 \\
0.72\end{array}$ \\
\hline 15 & 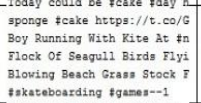 & $\begin{array}{l}\text { Top }=5 \\
\text { Top }=3\end{array}$ & & $\begin{array}{l}15 \\
10\end{array}$ & $\begin{array}{l}0.71 \\
0.83\end{array}$ \\
\hline 20 & 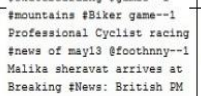 & $\begin{array}{l}\text { Top }=5 \\
\text { Top }=3\end{array}$ & & & $\begin{array}{l}0.78 \\
0.9\end{array}$ \\
\hline
\end{tabular}

Figure 10: Video-Topic relation on YouTube.

\subsection{Experimental Results and Analysis}

Within the experimental dataset, first we have randomly selected 25 active users and we have divided these users as new, light and heavy according to their video-related behavior on YouTube. The criterion for users is defined as, for the new user who does not have any video related behaviors, light users who have more than 7 videos related behaviors and for heavy users who have more than 10 video related interactions

and all the users have posted more than 70 tweets on his twitter accounts. Since the proposed solution is expected to be evaluated on three kinds of users new, light and heavy users, shown in Table No.1

Table 2: Video related interaction for three types of typical YouTube users.

\begin{tabular}{|c|c|c|c|c|}
\hline Dataset & Statistics & New User & Light User & Heavy User \\
\hline \multirow{3}{*}{ Train } & $\min$ & 0 & 7 & 10 \\
\cline { 2 - 5 } & avg & 0 & 8.3 & 12.5 \\
\cline { 2 - 5 } & $\max$ & 0 & 10 & 15 \\
\hline \multirow{3}{*}{ Test } & $\min$ & 20 & 20 & 21 \\
\cline { 2 - 5 } & $\operatorname{avg}$ & 23.23 & 23 & 26 \\
\cline { 2 - 5 } & $\max$ & 25 & 26 & 35 \\
\hline
\end{tabular}

\subsection{Comparison with Other Methods}

To evaluate the effectiveness of the proposed cross network recommendation solution on addressing the mentioned problems, i.e. New, light, heavy user, we have implemented three methods are listed as follows:
Word count based- Recommending videos to users by finding users preferences on twitter by calculating word count in each tweet document, it addresses new user problem.

Text similarity based - Recommending videos to users by calculating text similarity between each tweet document also we calculate similarity between video titles and recommend videos to new and light YouTube users.

Visual similarity based - Here we recommend videos to users by calculating the similarity between user uploaded videos with all other videos on YouTube on the basis of video frame features, it recommend videos to light and heavy users.

Cross Relevance based - Proposed method considers both twitter and YouTube OSNs and uses textual and visual similarity methods to recommend videos to three types of users; new, light, and heavy users on YouTube. It addresses the cold start and sparsity problems which have occurred in recommended systems. Proposed cross-network system recommends top 5 recommendation tasks and gives precision, recall, and F-score as an evaluation matrix, shown in Table No.2. For new user, system recommends top 5 videos with maximum word count on twitter. For light and heavy users, system recommends top videos by calculating user interest on twitter and YouTube using text and visual similarity based methods.

From the above-mentioned methods, text similarity and word count based methods are helpful to solve the new user problem. But, word count based method is effective as compared to text similarity. The visual similarity based method solves the cold start and sparsity problems. It helps to recommend videos to light and heavy users only. Among these methods our proposed cross-relevance based method solve three main problems in recommended systems and gives personalize video recommendation to new, light and heavy YouTube users, shown in Fig No. 11, 12, and 13.

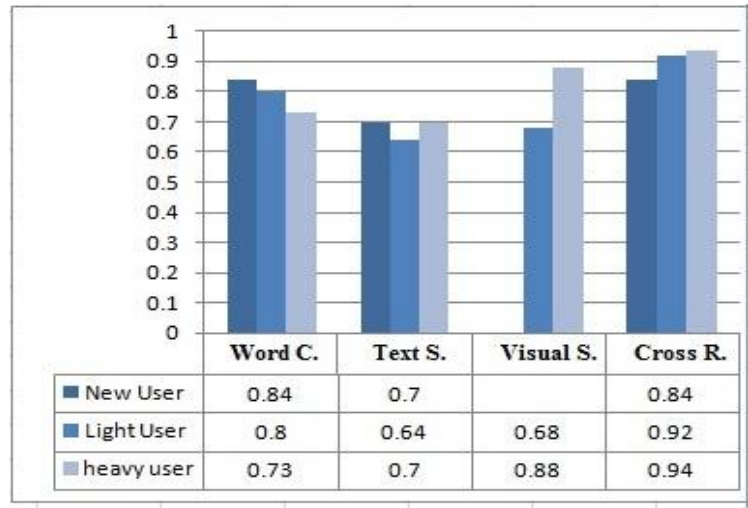

Figure 11: Top 5 precision values for three types of users using four different methods. 
Table 3: Top-5 precision, recall, and F-score for the examined methods on three test user sets.

\begin{tabular}{|c|c|c|c|c|c|}
\hline Test Set & Metrics & $\begin{array}{c}\text { Word Count } \\
\text { based }\end{array}$ & $\begin{array}{c}\text { Text } \\
\text { Similarity-based }\end{array}$ & $\begin{array}{c}\text { Visual } \\
\text { Similarity-based }\end{array}$ & $\begin{array}{c}\text { Cross-Relevance } \\
\text { based }\end{array}$ \\
\hline \multirow{3}{*}{ New User } & Precision & 0.84 & 0.7 & - & 0.84 \\
\hline & Recall & 0.7 & 0.42 & - & 0.72 \\
\hline & F-Score & 0.6 & 0.52 & - & 0.77 \\
\hline \multirow{3}{*}{ Light User } & Precision & 0.80 & 0.64 & 0.68 & 0.92 \\
\hline & Recall & 0.62 & 0.42 & 0.40 & 0.83 \\
\hline & F-Score & 0.69 & 0.50 & 0.50 & 0.87 \\
\hline \multirow{3}{*}{ Heavy User } & Precision & 0.73 & 0.7 & 0.88 & 0.94 \\
\hline & Recall & 0.48 & 0.41 & 0.69 & 0.89 \\
\hline & F-Score & 0.58 & 0.51 & 0.77 & 0.91 \\
\hline
\end{tabular}

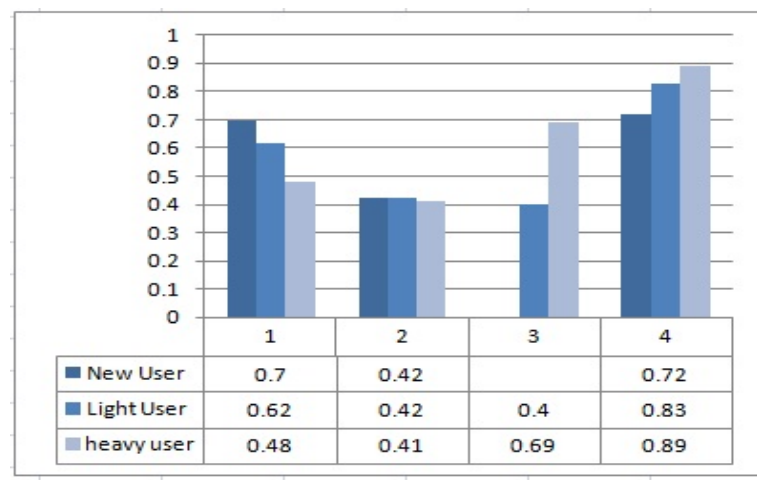

Figure 12: Top 5 Recall values for three types of users using four different methods.

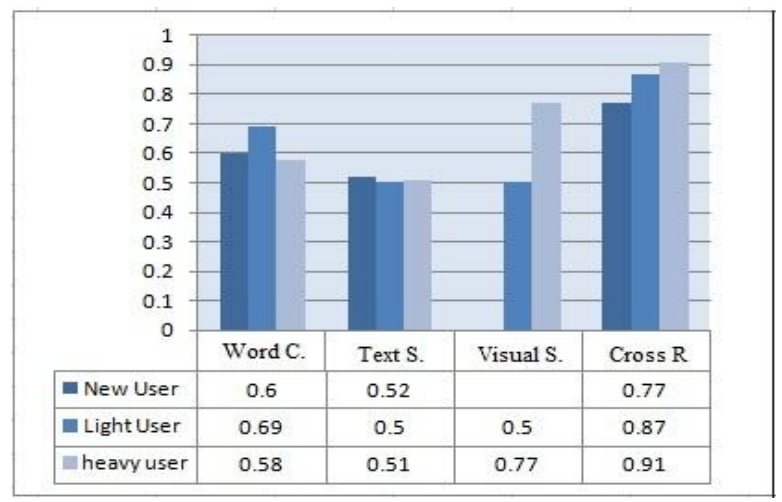

Figure 13: Top 5 F-score values for three types of users using four different methods.

\section{CONCLUSION AND FUTURE WORK}

The Cross-network YouTube recommendation system recommends videos to users based on their recent activities in different OSNs and gives benefit to three types of YouTube users. For new user system recommends videos, by analyzing user's recent tweet activities on Twitter. For light and heavy users, system recommends videos by integrating user's behavior on Twitter with observed video interaction on
YouTube, i.e. system constructs text and visual similarity between tweets and videos. The system finds out users preferences and likewise recommends videos to the users on YouTube. Here cross-Relevance method addresses new, coldstart, and sparsity problem occur in YouTube recommendation systems.

Our proposed system gives personalized video recommendation; by extracting user's data from their own auxiliary networks. But in future, we can also give a recommendation to these users by exploiting their social relational information from his/her twitter, YouTube, Google+ friend's network.

\section{ACKNOWLEDGMENT}

I am glad to express my feelings of gratitude to all who rendered their valuable guidance to me. I would also love to thank my guide Prof. Mrs. S. A. Takale for her guidance. I would like to express my gratefulness and thanks to the Principal of our college. I am also thankful to the Head of Department.

\section{REFERENCES}

[1] Zhengyu Deng, Jita Sang, and Changsheng Xu, "Unified YouTube Video Recommendation via Cross network Collaboration," June 23-26, 2015.

[2] http://nlprweb.ia.ac.cn/mmc/homepage/myan/Project_Ya nMing/ming_ICMR2015/crossnetwork\%20video\%20rec ommendation.html.

[3] Mi Zhang, Jie Tang, "Addressing Cold Start in Recommender Systems: A Semi-supervised Co-training Algorithm,'In Shanghai Key Laboratory of Intelligent Information Processing, Copyright 2014 ACM 978-14503-2257-7/14/07, July 6-11, 2014.

[4] Reza Zafarani and Huan Liu, "Connecting users across social media sites: a behavioral-modeling approach," In ACM SIGKDD 2013, pages 41-49, ACM.

[5] Zhengyu Deng, Jitao Sang, "Personalized Video recommendation based on cross-platform user modeling," In ICME 2013, pages 1-6, IEEE. 
[6] Suman Deb Roy, Tao Mei, Wenjun Zeng, "Social transfer: cross-domain transfer learning from social streams for media application," In ACM Multimedia 2012, pages 649-658.

[7] ChanLe Wu, Ming Xie, "Solving the Sparsity Problem in Recommender Systems Using Association Retrieval, "In National Engineering Research Center for Multimedia Software, Wuhan, China, (C) 2011 ACADEMY PUBLISHER.

[8] James Davidson, Benjamin Liebald, "The YouTube video recommendation System," In Proceedings of the fourth ACM conference on Recommender systems, pages 293-296, 2010.

[9] Hao Ma, "Recommender systems with social regularization," In Proceedings of the fourth ACM international conference on Web search and data mining, pages 287-296, ACM, 2011.

[10] Fabian Abel, "Analyzing cross-system user modeling on the social web," In Web Engineering, pages 28-43, Springer, 2011.

[11] Zi-Ke Zhang, Chuang Liu, "Solving the cold-start problem in recommender systems with social tags," EPL (Euro physics Letters), 92(2):28002, 2010.

[12] James Davidson, Benjamin Liebald, "The YouTube video recommendation system," In Proceedings of the fourth ACM conference on Recommender systems, pages 293-296, ACM, 2010.

[13] Yehuda Koren, "Factorization meets the neighborhood: a Multifaceted collaborative filtering model," In ACM 2008. Pages426-434. 